\title{
Agreement Between Athlete-Recalled and Clinically Documented Concussion Histories in Former Collegiate Athletes
}

\author{
Zachary Y. Kerr, ${ }^{\star \dagger \neq \S \| ~ P h D, ~ M P H, ~ J a s o n ~ P . ~ M i h a l i k, ~}{ }^{\ddagger \S \uparrow ~ P h D, ~ A T C, ~ K e v i n ~ M . ~ G u s k i e w i c z, ~}{ }^{\ddagger \S \| \uparrow ~ P h D, ~ A T C, ~}$ \\ Wayne D. Rosamond, ${ }^{\dagger} \mathrm{PhD}$, Kelly R. Evenson, ${ }^{\dagger} \mathrm{PhD}$, and Stephen W. Marshall, ${ }^{\dagger \neq \S \|} \mathrm{PhD}$ \\ Investigation performed at the University of North Carolina at Chapel Hill, \\ Chapel Hill, North Carolina, USA
}

\begin{abstract}
Background: Athlete-recalled and clinically documented concussion histories have been used in research on former athletes, but both have limitations. Comparisons of these 2 types of concussion histories are needed to improve the accuracy of estimates of concussion history for future research and clinical care.
\end{abstract}

Purpose: To estimate the agreement between athlete-recalled and clinically documented concussion histories during college and to explore reasons for differences.

Study: Cohort study (diagnosis); Level of evidence, 3.

Methods: Athlete-recalled concussion histories were provided by a convenience sample of 130 former collegiate athletes using an online questionnaire, and they were individually linked to previously collected clinical data that tracked medically diagnosed concussions at the host institution from 1996 to 2012 . The intraclass correlation coefficient (ICC 2,1$)$ was used to assess agreement between athlete-recalled and clinically documented concussion histories. Descriptive analyses were performed to assess reasons for disagreement.

Results: Agreement between athlete-recalled and clinically documented concussion histories was low $\left(\mathrm{ICC}_{2,1}=0.21 ; 95 \%\right.$ confidence interval, 0.05-0.37), but it was higher for women $\left(\mathrm{ICC}_{2,1}=0.65\right.$; 95\% confidence interval, 0.44-0.79) and for athletes playing more recently (2005-2012; $I \mathrm{ICC}_{2,1}=0.39 ; 95 \%$ confidence interval, 0.01-0.67). Of the 53 athletes who self-reported college sports-related concussions, $40 \%$ believed that they sustained impacts that should have been diagnosed as concussions but were undetected, and $21 \%$ admitted nondisclosure of suspected concussions. Common reasons for nondisclosure included the following: did not think injury was serious enough (91\%), did not know it was a concussion (73\%), and did not want to leave the game/practice (73\%).

Conclusion: Given the low agreement between athlete-recalled and clinically documented concussion histories, methodologic research is needed to improve the quality of tools used to assess concussion histories in former athletes.

Keywords: concussion; injury; epidemiology; traumatic brain injury; concurrent validity

Up to 3.8 million concussions are estimated to occur in sports in the United States annually. ${ }^{18}$ Studies of current and former athletes suggest that recurrent concussion is associated with adverse health effects, including cognitive, neurobehavioral, and somatic symptomatology; slower recovery from concussion symptomatology; and earlier onset of negative mental health outcomes, including depression, mild cognitive impairment, and Alzheimer's disease. ${ }^{7,9-11,15}$ A methodologic weakness of the evidence base on the long-term effects of concussions in former athletes is the current absence of information regarding the validity of athlete-recalled data and clinical records to

The American Journal of Sports Medicine, Vol. XX, No. X DOI: $10.1177 / 0363546514562180$

(C) 2015 The Author(s) compile concussion histories. In addition, no studies have compared the comparative validity of these 2 methods in athletes. The validity of methods for determining a history of traumatic brain injury is infrequently studied, in part because obtaining a "gold-standard" measure of traumatic brain injury is difficult.

Clinically documented concussion reports from athletes' playing careers may be incomplete because of undisclosed or undiagnosed concussions (particularly concussions that are not related to sports) and thus may be insensitive for a complete concussion history. ${ }^{5}$ Variability in the clinical and research communities in skill and knowledge related to assessing concussion also affects detection sensitivity. ${ }^{1,8,23}$ Concepts of what constitutes a concussion have evolved over time. ${ }^{8}$ Previous concussions, particularly those without loss of consciousness and amnesia, have gone undetected. ${ }^{21}$ 
Athlete recall is also imperfect. Recent findings suggest that $35 \%$ to $62 \%$ of athletes underreport concussions to coaches and/or team medical staff members. ${ }^{16}$ Reasons for nondisclosure include athletes' not realizing they had sustained concussions, not believing concussions were serious enough to warrant disclosure, and not wanting to leave the game and let down their teams. ${ }^{4,17,20,22,27}$

An improved understanding of the detection capabilities of both athlete-recalled and clinically documented concussion histories will help develop more accurate estimates of concussion history for research involving former athletes and for clinical management of current athletes. In this study, we compared athlete-recalled and clinically documented concussion histories in a group of former collegiate athletes. Our specific aims were to (1) estimate the level of agreement between athlete-recalled and clinically documented concussion histories that occurred during college and (2) explore the potential reasons why athlete-recalled and clinically documented concussion histories may differ.

\section{METHODS}

We used data from former collegiate athletes who played at the University of North Carolina at Chapel Hill. Athleterecalled concussion histories were obtained through an online self-administered questionnaire and individually linked to existing previously collected clinical data for the same athletes for their complete collegiate playing careers. The Institutional Review Board at the University of North Carolina at Chapel Hill approved all aspects of this study.

\section{Convenience Sample of Questionnaire Respondents}

A link to the online self-administered questionnaire was sent to the e-mail addresses of the former collegiate athletes who played at the University of North Carolina at Chapel Hill between 1987 and 2012. Reminder e-mails were sent every other week throughout a 3-month data collection window. The invitation and questionnaire did not include information about this study's specific research questions. The alumni association provided a list of 3657 e-mail addresses, and we received responses from 797 respondents. We had no means of determining how many of our e-mails were read, nor could we verify that the e-mail addresses were validly associated with former athletes.

\section{Athlete-Recalled Concussion History}

The self-administered online Qualtrics questionnaire was based on the retired National Football League players cohort health survey ${ }^{15}$ and collected information on sports history, concussion history, current physical and mental health, and demographics. The Appendix (available online at http://ajsm.sagepub.com/supplemental) provides the questions related to concussion history.

Respondents reported the numbers of concussions they sustained during participation in high school, college, and professional (if applicable) sports. In addition, respondents also reported the number of non-sports-related concussions (eg, from a automobile accidents, falls, or violence). Concussions were defined as "occurring typically, but not necessarily, from a blow to the head followed by a variety of symptoms that may include any of the following: headache, dizziness, loss of balance, blurred vision, 'seeing stars,' feeling in a fog or slowed down, memory problems, poor concentration, nausea, or throwing-up." Respondents were informed that "getting 'knocked out' or being unconscious does not always occur with a concussion." This definition was adapted from one used in previous research. ${ }^{22}$

For sport-related concussions sustained during college, respondents identified the dates on which the injuries occurred. Because it may be unrealistic for respondents to remember precise concussion dates, we also requested the year in school at the time of injury and additional qualitative information that specified the circumstances of the injury (eg, mechanism of injury, specific competition during which injury occurred). The process was then repeated for non-sport-related concussions sustained during college.

Respondents were asked about impacts (ie, "any other hard hits, bell-ringers, or dings") they sustained during participation in college sports that they believed should have been diagnosed by team medical staff members as concussions but were not. Respondents then answered why they thought these potential concussions went undiagnosed, as well as the sources of information that helped them subsequently understand that these impacts may have been undiagnosed concussions. All respondents who reported that they did not disclose all college sportsrelated concussions were asked the reason for

*Address correspondence to Zachary Y. Kerr, PhD, MPH, NCAA Injury Surveillance Program, Datalys Center for Sports Injury Research and Prevention, 401 West Michigan Street, Suite 500, Indianapolis, IN 46202, USA (e-mail: zkerr@datalyscenter.org).

${ }^{\dagger}$ Department of Epidemiology, University of North Carolina at Chapel Hill, Chapel Hill, North Carolina, USA.

‡Injury Prevention Research Center, University of North Carolina at Chapel Hill, Chapel Hill, North Carolina, USA.

$\S$ Matthew A. Gfeller Sport-Related Traumatic Brain Injury Research Center, Department of Exercise and Sport Science, University of North Carolina at Chapel Hill, Chapel Hill, North Carolina, USA.

"Center for the Study of Retired Athletes, Department of Exercise and Sport Science, University of North Carolina at Chapel Hill, Chapel Hill, North Carolina, USA.

"Department of Exercise and Sport Science, University of North Carolina at Chapel Hill, Chapel Hill, North Carolina, USA.

One or more of the authors has declared the following potential conflict of interest or source of funding: The Matthew A. Gfeller Sport-Related Traumatic Brain Injury Research and the Center for the Study of Retired Athletes are funded by private donations and various grant-funding agencies interested in understanding the current and late-life consequences and benefits of sports participation. The Injury Prevention Research Center is partially supported by the National Center for Injury Prevention and Control to facilitate research that increases knowledge of the consequences of injuries and opportunities for injury prevention. S.W.M. is partly supported by grant R49-CE001495 from the Centers for Disease Control and Prevention to the University of North Carolina Injury Prevention Research Center. 
nondisclosure, using a closed-response list originating from McCrea et al. ${ }^{22}$

\section{Historical Records of Clinically Documented Concussions}

Beginning in 2001, our university required preseason baseline testing as part of an ongoing clinical program for sports including basketball, cheerleading, diving, field hockey, football, lacrosse, soccer, track and field pole vault, and wrestling. ${ }^{8,24}$ Data from pilot testing of procedures (before the mandate) were also available for some former athletes from these sports who played from 1996 to 2000. To maximize the probability that an athlete-recalled concussion would have been clinically documented, we limited our analysis to those respondents who underwent a preseason battery of neurocognitive and balance tests.

The protocol for this preseason baseline testing and postconcussion assessment remained the same across this time period. The preseason battery of neurocognitive and balance tests was used to provide a baseline for concussion assessment. During all school-sanctioned practices and competitions, team medical staff members were on site to attend to any injuries, included suspected concussions. Concussion diagnosis was based on the expertise of the team medical staff members, who relied on previously published definitions of concussion, ${ }^{8,12,24}$ symptoms and signs reported and exhibited by the athlete, and reports by medical staff members and other witnesses regarding the condition of the injured player. The team physician and other clinical staff members evaluated athletes suspected to have been concussed and repeated the battery of neurocognitive and balance tests. The results of the postconcussion battery were compared with baseline assessments to assist medical staff members in making return-to-play decisions. Concussed athletes would have to demonstrate a return to preseason levels before being cleared for graduated return to play. These clinical data were mined for the specific dates when concussions were sustained by student athletes who underwent baseline assessments.

Of the 797 respondents with complete questionnaire data, we identified 130 athletes with preseason clinical data available. Of these, 54 played at a time when preseason testing was required for their sports (2001 and onward), and 76 athletes played during pilot testing of preseason clinical procedures before 2001 .

\section{Linking Questionnaire Responses and Clinical Records}

We linked the 2 sources of concussion history (athlete recall and clinical records) using name, sport, birth date, and approximate date of injury. Because no previous literature exists regarding matching concussion data, we created 2 levels of matching criteria, which we refer to as "hard" and "soft." A match between the questionnaire data (ie, athlete-recalled concussion) and clinical data (ie, clinically documented concussion) was defined as hard when (1) the date on which a respondent athlete recalled sustaining the concussion was within 6 months of the date reported by the clinical data and within the same sport season or (2) the school year during which a respondent reported sustaining the concussion (ie, freshman, sophomore, junior, senior, fifth-year senior, or graduate school) matched the school year reported by the clinical data. Soft matches were the same as hard matches except that discrepancies between sources on the date of injury were allowable if there was agreement about the circumstances of injury (mechanism of injury and the specific competition during which the injury occurred).

\section{Statistical Analysis}

All analyzed data originated from the 130 former collegiate athletes who provided questionnaire data (to obtain athleterecalled concussion history) and had preseason clinical data available (to obtain clinically documented concussion history). The intraclass correlation coefficient $\left(\mathrm{ICC}_{2,1}\right)^{3}$ was used to assess agreement between athlete-recalled and clinically documented concussion histories. We also categorized concussions by those reported (1) only in athlete-recalled concussion history data, (2) only in clinically documented concussion history data, and (3) in both athlete-recalled and clinically documented concussion history data. We calculated the proportion of athlete-recalled concussions that were also clinically documented as the number of athlete-recalled concussions that were also clinically documented divided by the number of all athlete-recalled concussions. We then calculated the proportion of clinically documented concussions that were also athlete recalled as the number of clinically documented concussions that were also athlete recalled divided by the number of clinically documented concussions.

Results were also stratified by sex and level of contact. We categorized level of contact as collision sports (ie, football, wrestling; $n=42$ ), high-contact sports (ie, basketball, field hockey, lacrosse, and soccer; $n=79$ ), and low- or noncontact sports (ie, baseball, cheerleading, diving, rowing, and track and field; $n=9$ ).

Because changes in concussion reporting and awareness and diagnostic trends over time influence recall of concussions, we stratified results by the year in which athletes began playing collegiate sports: before $1996(\mathrm{n}=25)$, 1996-2000 $(\mathrm{n}=49), 2001-2004(\mathrm{n}=28)$, and 2005 and after $(n=28)$. Because clinical data were available only beginning with the 1996-1997 school year, we excluded any concussions respondents reported before the 1996-1997 school year $(n=2)$.

Finally, we tabulated counts of the items endorsed as the reasons respondents believed college sports-related concussions went undiagnosed and undisclosed (on the basis of the list of potential reasons we provided) and sources of information for concussion knowledge (on the basis of a list of potential sources we provided, such as the media, peers, and family and friends). Write-in responses for both questions were also tabulated.

\section{RESULTS}

The majority of the 130 former collegiate athletes with clinical data available were male $(63.1 \%[\mathrm{n}=82])$ and 
TABLE 1

Former Collegiate Athletes With

Clinical Data, by Sport $(\mathrm{n}=130)$

\begin{tabular}{lc}
\hline Sport & $\mathrm{n}(\%)$ \\
\hline Men's basketball & $5(3.8)$ \\
Men's cheerleading & $2(1.5)$ \\
Men's diving & $1(0.8)$ \\
Men's football & $32(24.6)$ \\
Men's lacrosse & $19(14.6)$ \\
Men's soccer & $11(8.5)$ \\
Men's track and field pole vault & $2(1.5)$ \\
Men's wrestling & $10(7.7)$ \\
Women's basketball & $4(3.1)$ \\
Women's field hockey & $8(6.2)$ \\
Women's lacrosse & $23(17.7)$ \\
Women's soccer & $9(6.9)$ \\
Women's track and field pole vault & $4(3.1)$ \\
Total & $130(100.0)$ \\
\hline
\end{tabular}

played before 2001 (57.7\% [ $\mathrm{n}=75]$ ). Most were non-Hispanic white $(83.8 \%[\mathrm{n}=109])$, followed by non-Hispanic black $(13.1 \%[n=17])$ and mixed race $(3.1 \%[n=4])$. The mean age was $30.9 \pm 4.4$ years. A diverse range of sports was included (Table 1). The mean time since the last year of collegiate play was $9.5 \pm 4.2$ years.

\section{Agreement Between Athlete-Recalled and Clinically Documented Concussions}

The mean number of athlete-recalled concussions per former collegiate athlete $(0.79 \pm 1.27)$ was nearly 3 times the mean number of clinically documented concussions per former collegiate athlete $(0.28 \pm 0.57)$. Athlete-recalled concussion history indicated that $43.8 \%(\mathrm{n}=57)$ had sustained 1 or more concussions during college, of whom 53 (40.8\%) sustained concussions during participation in collegiate sports. In contrast, clinically documented concussion history indicated that $22.3 \%(\mathrm{n}=29)$ had sustained 1 or more concussions during college. Of these, only 2 $(6.9 \%)$ had clinically documented non-sport-related concussions.

Agreement between the number of athlete-recalled concussions and clinically documented concussions was low $\left(\mathrm{ICC}_{2,1}=0.21 ; 95 \%\right.$ confidence interval $[\mathrm{CI}], 0.05-0.37$; Table 2). There were 21 hard matches, accounting for $58.3 \%$ of the 36 clinically documented concussions and $20.4 \%$ of the 103 athlete-recalled concussions (Table 3). In other words, on the basis of hard matches alone, athletes failed to recall $41.7 \%$ of those concussions that were clinically documented, and there were no clinical data associated with $79.6 \%$ of athlete-recalled concussions. Most of these hard matches had self-reported and clinically documented dates within 1 month; the largest difference between dates was 4 months. In addition, 4 soft matches were generated from information on circumstances of injury (obtained from both respondents and clinical data); 3 noted the same competition during which the injury occurred, and 1 noted the same mechanism of injury. All of the athlete-reported dates of these soft matches were off from the clinically documented dates by 1 year (eg, athlete-reported date of May 2005 and clinically documented date of May 2006). Inclusion of the soft matches increased the number of matches to 25 , accounting for $69.4 \%$ of clinically documented concussions and $23.4 \%$ of athlete-recalled concussions. In other words, after including the soft matches, athletes failed to recall $31.6 \%$ of those concussions that were clinically documented, and there were no clinical data associated with $76.6 \%$ of athlete-recalled concussions.

Findings were stratified by sex, level of contact in sport, and year began playing collegiate sports. In all strata, the average number of athlete-recalled concussions per former collegiate athlete was higher than that of clinically documented concussions (Table 2). The $\mathrm{ICC}_{2,1}$ for women $(0.65 ; 95 \%$ CI, 0.44-0.79) was higher than that for men (0.13; 95\% CI, 0.00-0.31). Compared with women (36.8\%), men had a larger percentage of clinically documented concussions that were athlete recalled (82.4\%) (Table 3). In addition, the perentage of athlete-recalled concussions that had associated clinical data were highest among Hispanic nonwhite athletes $(33.3 \%)$ and those whose collegiate sports careers began in 2005 and after $(32.0 \%)$.

\section{Reported Reasons for Nondisclosure and Sources of Information on Concussion}

Of the 53 former collegiate athletes reporting college sports-related concussions, 21 (39.6\%) reported that they believed they had college sports-related concussions that went undiagnosed. Common self-reported reasons for nondiagnosis were that respondents did not tell team medical staff members $(\mathrm{n}=11)$ and that no team medical staff members were present $(n=4)$. The 11 respondents who did not disclose all their sports-related concussions to team medical staff members provided numerous reasons for nondisclosure, including not thinking it was serious enough $(90.9 \%[\mathrm{n}=10])$, not knowing it was a concussion $(90.9 \%[n=10])$, not wanting to leave the game or practice $(90.9 \%[\mathrm{n}=10])$, not wanting to let the team down $(63.6 \%$ $[\mathrm{n}=7])$, and not wanting to be pulled from future games or practices $(54.5 \%[\mathrm{n}=6])$. All 21 respondents noted in text responses that recent concussion knowledge had helped them realize that these impacts may have been undiagnosed concussions. Sources of knowledge included friends $(23.8 \%[n=5])$, continuing education $(23.8[n=5])$, and the media $(85.7 \%[\mathrm{n}=18])$, particularly ESPN $(42.9 \%$ [n $=9]$ ), other television shows $(28.6 \%[n=6])$, and research $\operatorname{articles}(14.3 \%[\mathrm{n}=3])$.

\section{DISCUSSION}

Obtaining accurate concussion histories from former athletes is an important measurement issue in studies of associations between recurrent concussion and negative health outcomes such as depression and mild cognitive impairment. ${ }^{7,9,10,15}$ Clinically documented concussion histories are limited because medical records are inconsistent, hard to access, or nonexistent. Athlete-recalled concussion 
TABLE 2

Number of Athlete-Recalled and Clinically Documented Concussions in Former Collegiate Athletes, by Sex, Level of Contact in Sport, and Time ${ }^{a}$

\begin{tabular}{|c|c|c|c|c|}
\hline Variable & Athletes, No. (\%) & $\begin{array}{l}\text { Athlete-Recalled Concussions } \\
\text { per Former Athlete, Mean } \pm \mathrm{SD}^{b}\end{array}$ & $\begin{array}{l}\text { Clinically Documented Concussions } \\
\text { per Former Athlete, Mean } \pm \mathrm{SD}^{c}\end{array}$ & $\mathrm{ICC}_{2,1}(95 \% \mathrm{CI})$ \\
\hline Total & $130(100.0)$ & $0.79 \pm 1.27$ & $0.28 \pm 0.57$ & $0.21(0.05-0.37)$ \\
\hline Male & $82(63.1)$ & $0.90 \pm 1.47$ & $0.21 \pm 0.49$ & $0.13(0.00-0.31)$ \\
\hline Female & $48(36.9)$ & $0.58 \pm 0.79$ & $0.40 \pm 0.68$ & $0.65(0.44-0.79)$ \\
\hline \multicolumn{5}{|c|}{ Level of contact in sport } \\
\hline Collision & $42(32.3)$ & $0.79 \pm 1.28$ & $0.12 \pm 0.33$ & $0.05(0.00-0.30)$ \\
\hline \multicolumn{5}{|c|}{ Year began collegiate sports } \\
\hline Before 1996 & $25(19.2)$ & $1.04 \pm 1.77$ & $0.16 \pm 0.47$ & $0.08(0.00-0.42)$ \\
\hline $1996-2000$ & $49(37.7)$ & $0.71 \pm 1.31$ & $0.31 \pm 0.51$ & $0.24(0.00-0.48)$ \\
\hline $2001-2004$ & $28(21.5)$ & $0.57 \pm 0.84$ & $0.32 \pm 0.77$ & $0.33(0.00-0.62)$ \\
\hline 2005 and after & $28(21.5)$ & $0.89 \pm 1.03$ & $0.29 \pm 0.53$ & $0.39(0.01-0.67)$ \\
\hline
\end{tabular}

${ }^{a} \mathrm{CI}$, confidence interval; $\mathrm{ICC}_{2,1}=$ intraclass correlation coefficient.

${ }^{b}$ Athlete-recalled concussion history originated from online questionnaire.

${ }^{c}$ Clinically documented concussion history originated from clinical data.

TABLE 3

Distribution of Athlete-Recalled and Clinically Documented Concussions in Former Collegiate Athletes, by Sex, Level of Contact in Sport, and Time ${ }^{a}$

\begin{tabular}{|c|c|c|c|c|c|c|c|}
\hline \multirow[b]{2}{*}{ Variable } & \multicolumn{3}{|c|}{ Concussions, No. } & \multicolumn{2}{|c|}{$\begin{array}{c}\text { Athlete-Recalled Concussions } \\
\text { That Were Clinically } \\
\text { Documented, \% }\end{array}$} & \multicolumn{2}{|c|}{$\begin{array}{c}\text { Clinically Documented } \\
\text { Concussions That Wer } \\
\text { Athlete-Recalled, \% } \\
\end{array}$} \\
\hline & $\begin{array}{l}\text { Both Athlete- } \\
\text { Recalled and } \\
\text { Clinically } \\
\text { Documented }\end{array}$ & $\begin{array}{l}\text { Athlete-Recalled } \\
\text { but Not } \\
\text { Clinically } \\
\text { Documented }\end{array}$ & $\begin{array}{l}\text { Clinically } \\
\text { Documented but } \\
\text { Not Athlete- } \\
\text { Recalled }\end{array}$ & $\begin{array}{l}\text { Hard } \\
\text { Matches } \\
\text { Only }\end{array}$ & $\begin{array}{l}\text { With Soft } \\
\text { Matches }\end{array}$ & $\begin{array}{l}\text { Hard } \\
\text { Matches } \\
\text { Only }\end{array}$ & $\begin{array}{c}\text { With Soft } \\
\text { Matches }\end{array}$ \\
\hline Total & 21 & 82 & 15 & 20.4 & 23.4 & 58.3 & 69.4 \\
\hline \multicolumn{8}{|l|}{ Sex } \\
\hline Male & 14 & 59 & 3 & 19.2 & 19.2 & 82.4 & 82.4 \\
\hline Female & 7 & 23 & 12 & 23.3 & 32.4 & 36.8 & 57.9 \\
\hline \multicolumn{8}{|c|}{ Level of contact in sport } \\
\hline Low/none & 0 & 5 & 0 & 0.0 & 0.0 & $-^{b}$ & ${ }^{b}$ \\
\hline High & 16 & 51 & 15 & 23.9 & 28.2 & 51.6 & 64.5 \\
\hline Collision & 5 & 26 & 0 & 16.1 & 16.1 & 100.0 & 100.0 \\
\hline \multicolumn{8}{|c|}{ Year began collegiate sports } \\
\hline Before 1996 & 2 & 22 & 3 & 8.3 & 12.0 & 40.0 & 60.0 \\
\hline $1996-2000$ & 8 & 29 & 6 & 21.6 & 23.7 & 57.1 & 64.3 \\
\hline $2001-2004$ & 3 & 13 & 6 & 18.8 & 27.8 & 33.3 & 55.6 \\
\hline 2005 and after & 8 & 17 & 0 & 32.0 & 32.0 & 100.0 & 100.0 \\
\hline
\end{tabular}

${ }^{a}$ Athlete-recalled concussion history originated from questionnaire data; clinically documented concussion history originated from clinical data. Hard matches $(n=21)$ were defined as those for which (1) the date on which a respondent athlete recalled sustaining the concussion was within 6 months and within the same season as the date reported in the clinical data or (2) the school year that a respondent reported sustaining the concussion matched the school year reported by the clinical data. Soft matches $(n=4)$ did not adhere to criteria for matching athlete-recalled and clinically documented concussions but could still be possible matches, given additional qualitative information provided by respondents and clinical data about the circumstances of injury to plausibly link records (eg, mechanism of injury, specific competition during which injury occurred).

${ }^{b}$ There were no clinically documented concussions for the "low/none" level of contact in sport.

histories are prone to potential recall effects and other subjective information that lacks clinical validation. This is the first study to compare athlete-recalled concussion histories with clinically documented concussion history data in an attempt to study whether the 2 methods yield comparable results. 
Athlete-recalled and clinically documented concussion histories had low agreement $\left(\mathrm{ICC}_{2,1}=0.21\right)$. Four of 5 athleterecalled concussions were not corroborated by clinical data. The only exception was for clinically documented concussions from 2005 onward; former athletes recalled all these concussions. However, it is unclear if the better agreement $\left(\mathrm{ICC}_{2,1}=\right.$ 0.39 ) was due to improved clinical detection in this era, better recall for more recent concussions, or a combination of both. It is also important to consider that our sample of former collegiate athletes with questionnaire and clinical data was small $(\mathrm{n}=130)$, and we were also unable to obtain preseason clinical records for $19 \%$ of individuals $(n=13)$. Nevertheless, the minimal overlap between the 2 methods for assessing concussion history suggests that neither athlete recall nor clinical documentation currently serves as a suitable gold standard.

Our method for obtaining athlete-recalled concussion history was an online self-administered questionnaire. However, results may have differed had we used other methods to obtain athlete-recalled concussion history. A face-to-face interview would allow researchers to obtain details on athletic and nonathletic life events that could be used as cognitive anchors to facilitate better recall of concussive symptoms. Additionally, education about concussion symptomatology may have helped respondents provide accurate histories.

We were also unable to verify that athlete-recalled concussions met the clinical definition of concussion used by medical professionals. Thus, former athletes may have retrospectively perceived subconcussive impacts or cervical injuries to be concussions. ${ }^{19}$ For example, concussions may cause symptoms similar to other sports medicine disorders (eg, overexertion and dehydration). ${ }^{25}$ It is also possible that past concussions were also misdiagnosed because of the more restricted understanding of what constitutes a concussion. Nevertheless, given current concussion management requirements, it is highly unlikely that sports medicine professionals would not communicate concussion diagnoses to athletes. The fact participants from 2005 and later recalled all of their clinically diagnosed concussions suggests that communication between clinicians and athletes about the diagnosis of a concussion is not a highly relevant issue at the present time.

However, we also caution that clinical practice and understanding of concussion (eg, clinical management, diagnosis, education) have evolved significantly during the period of time during which our cohort played collegiate sports. Some concussions that were not identified by clinical staff members in a previous era might be reported today, because of improvements in clinician training and heightened awareness of concussion. Further to this point, there may have been better agreement in more recent years because of improved diagnostic criteria and more complete recall by athletes. Because of its evolution, a strict definition of concussion was not used. Team clinical staff members relied on previously published definitions of concussion, ${ }^{8,12,24}$ symptoms and signs reported and exhibited by the athlete, and reports by medical staff members and other witnesses regarding the condition of the injured player. It is also possible that current clinical practice may still potentially miss some concussions and may heavily rely on athletes to state that they feel symptomatic to initiate concussion management protocols. Future research seeking to better understand the concurrent validity of self-reported and clinically documented concussion histories may benefit from using more recent concussion data, in which concussions are based on physical findings and neurocognitive testing. Because there is also much interest in the effects of long-ago concussions on retired athletes, research should also explore how athletic trainers and physicians' knowledge of concussions have changed over time, so that we better ascertain the proportion of concussions that clinically documented histories may have previously missed.

Women had better agreement between athlete-recalled and clinically documented concussion histories than men. Previous findings have noted that men were more likely to not report symptoms of concussion to coaches or athletic training staff members,${ }^{28}$ and impacts sustained by women may be more likely diagnosed as concussions. ${ }^{6}$ This would explain the higher average number of clinically documented concussions for women compared with men. However, a previous study ${ }^{20}$ found no differences in concussion disclosure between men and women. As concussion-related knowledge increases, there is potential for better exploration of sex differences related to the incidence and reporting of concussions.

Nearly half of the respondents believed that they had sustained impacts that should have been diagnosed as concussions at the time of injury. Close to half noted nondisclosure, particularly subscribing to reasons noted in previous studies, such as lack of knowledge and pressure to play while concussed. ${ }^{4,17,20,22,27}$ Preseason educational and social norming interventions that address nondisclosure may help athletes more willingly disclose suspected concussions and symptoms, thus helping sports organizations more effectively detect and manage concussions.

Respondents also reported that undiagnosed concussions occurred because team medical staff members were not present at the time of injury. Other studies have noted the lack of medical staff presence at sports settings, ${ }^{26}$ even when they were on staff. ${ }^{13}$ A high level of staffing at all practices and competitions will help ensure that all injuries sustained by student athletes and staff are identified and treated and managed in a timely manner.

Information from peers and the media might have assisted respondents in increasing their concussion-related knowledge, and thus, they may have retrospectively reconsidered previously sustained impacts. Thus, our results are further complicated by the external sources potentially influencing concussion recall. ${ }^{14,16}$ Such changes in perception are consistent with social cognitive theory, which states that individuals acquire knowledge through the observation of others via social contexts, media, and other modes of communication. ${ }^{2}$ As a result, gaining new knowledge of concussions when participants may not have previously known they had been concussed could help explain some of the differences between diagnosed and undiagnosed concussions. 


\section{Limitations}

No gold standard of concussion history exists to evaluate current methods' acquisition of concussion frequency. Although the concussion definition used at the University of North Carolina at Chapel Hill would ideally have remained constant through the study period, in reality, there was considerable evolution in the detection and management of concussion nationally over the time these respondents were college athletes. Therefore, we may have heterogeneity in our sample due to improvements in clinical staff members' detection and diagnoses of clinically documented concussions over time. Furthermore, not all concussions evaluated outside of the university setting may have been documented, particularly those occurring during summer or winter break. We also lacked clinical data from outside of the collegiate setting (eg, high school and professional levels).

Only 130 former collegiate athletes had clinical data available, all were recruited from 1 university, and all played between 1987 and 2012. Thus, our findings may not be generalizable to nonrespondents, respondents without clinical data, and former athlete populations from other universities, playing eras, or playing levels (eg, high school). In particular, it is possible that former collegiate athletes who may have retrospectively thought they had undiagnosed concussions may have been more likely to respond to our questionnaire. To minimize this potential bias, our study's invitation to participate and questionnaire did not mention this study's specific research questions.

The findings are also likely not generalizable to the current time period given the small number of responses from former collegiate athletes who began their sports careers in 2005 and after, who played during a time when there was a rapidly changing culture and policies and procedures with respect to concussions. We chose to limit our group to those 130 athletes with preseason baseline tests, and this is a further source of nongeneralizability. This practice was uncommon during the 1980s and 1990s and was limited to certain sports in the 2000s. Furthermore, this higher level of concussion surveillance and management may have potentially discouraged athletes from reporting.

\section{CONCLUSION}

Our findings suggest that agreement between athleterecalled and clinically documented concussion histories is low. Each source of concussion history data failed to account for large proportions of concussions identified by the other data source. However, higher agreement was found among former athletes who played collegiate sports more recently. This may suggest improved clinical detection in recent years, less recall decay of recent events, or both. External influences such as peers and the media may influence former athletes' reporting of concussion histories. Future studies with former athletes must consider the strengths and limitations of each data source. Significant methodological innovation is recommended to improve the quality of concussion reporting questionnaires and documentation in medical records. This may include using athletic and nonathletic life events to help facilitate better recall of concussive symptoms cognitive anchors and/or using sensor data combined with self-reports to better detect concussions.

\section{ACKNOWLEDGMENT}

The authors thank the staff members and students affiliated with both centers for their assistance in this research study.

\section{REFERENCES}

1. Aubry M, Cantu R, Dvorak J, et al. Summary and agreement statement of the First International Conference on Concussion in Sport, Vienna 2001: recommendations for the improvement of safety and health of athletes who may suffer concussive injuries. $\mathrm{Br} J$ Sports Med. 2002;36(1):6-10.

2. Bandura A. Social Foundations of Thought and Action: A Social Cognitive Theory. Englewood Cliffs, NJ: Prentice Hall; 1986.

3. Bartko JJ. The intraclass correlation coefficient as a measure of reliability. Psychol Rep. 1966;19(1):3-11.

4. Broglio SP, Vagnozzi R, Sabin M, et al. Concussion occurrence and knowledge in Italian football (soccer). J Sports Sci Med. 2010;9(3):418-430.

5. Collins MW, Lovell MR, Iverson GL, et al. Cumulative effects of concussion in high school athletes. Neurosurgery. 2002;51(5):1175-1179.

6. Dick R. Is there a gender difference in concussion incidence and outcomes? Br J Sports Med. 2009;43(Suppl 1):i46-i50.

7. Didehbani N, Cullum CM, Mansinghani S, et al. Depressive symptoms and concussions in aging retired NFL players. Arch Clin Neuropsychol. 2013;28(5):418-424.

8. Guskiewicz KM, Bruce SL, Cantu RC, et al. National Athletic Trainers' Association position statement: management of sport-related concussion. J Athl Train. 2004;39(3):280-297.

9. Guskiewicz KM, Marshall SW, Bailes J, et al. Association between recurrent concussion and late-life cognitive impairment in retired professional football players. Neurosurgery. 2005;57(4):719-726.

10. Guskiewicz KM, Marshall SW, Bailes J, et al. Recurrent concussion and risk of depression in retired professional football players. Med Sci Sports Exerc. 2007;39(6):903-909.

11. Guskiewicz KM, McCrea M, Marshall SW, et al. Cumulative effects associated with recurrent concussion in collegiate football players: the NCAA Concussion Study. JAMA. 2003;290(19):2549-2555.

12. Kelly JP, Rosenberg JH. Diagnosis and management of concussion in sports. Neurology. 1997;48(3):575-580.

13. Kerr ZY, Casa DJ, Marshall SW, et al. Epidemiology of exertional heat illness among U.S. high school athletes. Am J Prev Med. 2013;44(1):8-14.

14. Kerr ZY, Marshall SW, Guskiewicz KM. Reliability of concussion history in former professional football players. Med Sci Sports Exerc. 2012;44(3):377-382.

15. Kerr ZY, Marshall SW, Harding HP, et al. Nine-year risk of depression diagnosis increases with increasing self-reported concussions in retired professional football players. $A m$ J Sports Med. 2012;40(10):2206-2212.

16. Kerr ZY, Register-Mihalik JK, Marshall SW, et al. Disclosure and nondisclosure of concussion and concussion symptoms in athletes: review and application of the socio-ecological framework. Brain Injury. 2014;28(8):1009-1021.

17. Kroshus E, Daneshvar DH, Baugh CM, et al. NCAA concussion education in ice hockey: an ineffective mandate. $\mathrm{Br} J$ Sports Med. 2014;48(2):135-140. 
18. Langlois JA, Rutland-Brown W, Wald MM. The epidemiology and impact of traumatic brain injury: a brief overview. J Head Trauma Rehabil. 2006;21(5):375-378.

19. Leddy JJ, Baker JG, Merchant A, et al. Brain or strain? Symptoms alone do not distinguish physiologic concussion from cervical/vestibular injury [published online July 21, 2014]. Clin J Sport Med. doi:10.1097/ JSM.0000000000000128.

20. Llewellyn T, Burdette GT, Joyner AB, et al. Concussion reporting rates at the conclusion of an intercollegiate athletic career. Clin $J$ Sport Med. 2014;24(1):76-79.

21. McCrea M, Guskiewicz KM, Marshall SW, et al. Acute effects and recovery time following concussion in collegiate football players: the NCAA Concussion Study. JAMA. 2003;290(19):2556-2563.

22. McCrea M, Hammeke T, Olsen G, et al. Unreported concussion in high school football players: implications for prevention. Clin J Sport Med. 2004;14(1):13-17.

23. McCrory P, Johnston K, Meeuwisse W, et al. Summary and agreement statement of the 2nd International Conference on
Concussion in Sport, Prague 2004. Br J Sports Med. 2005;39(4): 196-204.

24. McCrory P, Meeuwisse W, Johnston K, et al. Consensus statement on concussion in sport: the 3rd International Conference on Concussion in Sport held in Zurich, November 2008. J Athl Train. 2009;4(4):434-448.

25. Mueller FO, Casa DJ. Fatal and catastrophic injuries in athletes: Epidemiologic data and challenging circumstances. In Casa DJ, ed. Preventing Sudden Death in Sport and Physical Activity. Sudbury, Massachusetts: Jones and Bartlett Learning; 2012:1-14.

26. National Athletic Trainers' Association. Athletic trainers fill a necessary niche in secondary schools. Available at: http://www.nata.org/ NR031209. Accessed February 20, 2014.

27. Register-Mihalik JK, Guskiewicz KM, McLeod TC, et al. Knowledge, attitude, and concussion-reporting behaviors among high school athletes: a preliminary study. J Athl Train. 2013;48(5):645-653.

28. Torres DM, Galetta KM, Phillips HW, et al. Sports-related concussion: anonymous survey of a collegiate cohort. Neurol Clin Pract. 2013;3(4):279-287.

For reprints and permission queries, please visit SAGE's Web site at http://www.sagepub.com/journalsPermissions.nav 